\title{
OIL SHALE PHENOL-DERIVED AEROGELS AS SUPPORTS FOR PALLADIUM NANOPARTICLES
}

\author{
F. PÉREZ-CABALLERO ${ }^{(a) *}$, A.-L. PEIKOLAINEN ${ }^{(a)}$, \\ M. UIBU ${ }^{(b)}$, M. HERBERT ${ }^{(c)}$, A. GALINDO ${ }^{(c)}$, \\ F. MONTILLA ${ }^{(\mathrm{c})}$, M. KOEL ${ }^{(a)}$ \\ (a) Institute of Chemistry, Tallinn University of Technology, Akadeemia tee 15, \\ 12618 Tallinn, Estonia \\ (b) Laboratory of Inorganic materials, Tallinn University of Technology, Ehitajate \\ tee 5, 19086 Tallinn, Estonia \\ (c) Departamento de Quimica Inorginica, Universidad de Sevilla, Aptdo. 1203, \\ 41071 Sevilla, Spain
}

\begin{abstract}
Palladium hexafluoroacetylacetonate $\left[\mathrm{Pd}\left(\mathrm{C}_{4} \mathrm{HF}_{6} \mathrm{O}\right)_{2}\right]$, which is both soluble and easily reducible in supercritical carbon dioxide, was used to impregnate the porous structure of carbon aerogels based on phenolic compounds from oil shale processing water. Metallic nanoparticles uniformly distributed on the surface of the carbon matrix enable it to be used as a catalyst. Through the study of various pyrolysis programs and physical activation with $\mathrm{CO}_{2}$ and $\mathrm{H}_{2} \mathrm{O}$ in the carbon aerogel preparation process, significant increases in the BET specific surface area of the carbon supports (achieving values of over $1600 \mathrm{~m}^{2} / \mathrm{g}$ ) were achieved, and the possibility of tailoring the porosity of the samples (microporosity ranging from $40 \%$ up to over 90\%) was realized.
\end{abstract}

\section{Introduction}

The common raw material for carbon aerogels is organic aerogel pyrolysed under an inert atmosphere at high temperatures [1]. Pekala et al. first reported synthesis of organic aerogels by sol-gel polycondensation of resorcinol (1,3-dihydroxybenzene) and formaldehyde, and subsequent drying using supercritical carbon dioxide [2,3]. In our laboratory the efforts are made to prepare aerogels using phenolic compounds from oil shale industry.

In the case of using 5-methylresorcinol, it was shown that a much shorter preparation time as well as a stiffer structure due to the additional directing methyl group were advantageous compared to the use of resorcinol [4]. It was demonstrated that an industrial mixture of phenols - Honeyol ${ }^{\circledR}$ can be

\footnotetext{
* Corresponding author: e-mail fernanlora@ hotmail.com
} 
successfully used for obtaining aerogels [5]. These carbon aerogels have high porosity (over 50\%) and high surface areas $\left(400-1000 \mathrm{~m}^{2} \mathrm{~g}^{-1}\right.$ ) which can be increased by a simple activation after pyrolysis as it is investigated in this research.

The first goal of this work was to optimize the process of pyrolysis of organic aerogel to obtain porous carbon material with proper parameters for further impregnation.

One must consider that during pyrolysis, carbon aerogels undergo mass loss leading to volumetric shrinkage and densification compared to their precarbonized counterparts [6-8].

Also, a study on the activation of carbon aerogels (CAs) was carried out. Activation of a carbon material is a process to obtain very high specific surface area and to increase the pore volume of CAs [9]. In physical activation, steam and carbon dioxide are generally preferred in the gasification step, during which slow gasification kinetics allows gas molecules to diffuse into the carbon pores and develop a large surface area. The BET surface area of $3200 \mathrm{~m}^{2} / \mathrm{g}$ is the highest value that is expected for carbon aerogels [10]. Following the pyrolysis, changes in pore structures during further gasification are monitored and controlled. Activation improves pore volume and surface area of CAs. Therefore, the activation would be helpful for the application of CAs as absorbents [11].

Nanoparticles on different support materials are currently used extensively as catalysts for chemical transformation. The surface where the nanoparticles are placed plays an important role in the final catalytic properties [12].

The second goal of our work was to produce homogeneous palladiumdoped carbon aerogels in order to study their catalytic activity.

To impregnate the carbon network with metal nanoparticles, several methods have been developed, most beginning with an oxidized salt or metal complex followed by reduction and decompression [13,14]. A number of methods for reduction of the metal have been employed including thermal decomposition in an inert atmosphere and chemical conversion with hydrogen or air after depressurization [15]. The most advanced method is the impregnation of porous material and following reduction in the supercritical fluid (SCF) with a reducing agent such as hydrogen or an alcohol and thermal reduction in the supercritical fluid [16].

The SCF medium allows an easy penetration and wetting of pores compared to normal solvents, as well as avoiding pore collapses that occur when an aerogel is dried by leaving the sample to dry under ambient conditions. Amongst commonly used SCFs, supercritical carbon dioxide $\left(\mathrm{scCO}_{2}\right)$ is characterized by easily accessible supercritical parameters $\left(\mathrm{T}_{\mathrm{c}}=31.1{ }^{\circ} \mathrm{C} ; \mathrm{P}_{\mathrm{c}}=7.38 \mathrm{MPa}\right)$, is non-flammable, non-toxic, environmentally friendly, inexpensive and leaves no residues in the treated medium [17]. 


\section{Materials and methods}

5-Methylresorcinol with a reported purity of $99.58 \%$ was obtained from Carboshale AS and Honeyol ${ }^{\circledR}$ containing 56\% 5-methylresorcinol was obtained from VKG AS, these materials were was used as received. Anhydrous sodium carbonate with a purity of $99.8 \%$ from Riedel-de-Haen (Germany) was used as received. Water used was purified using Milli-Q Water system. Formaldehyde $(37 \% \mathrm{w} / \mathrm{w}$ solution in water) was obtained from Aldrich and was used as received. Finally, the complex $\left[\mathrm{Pd}\left(\mathrm{C}_{4} \mathrm{HF}_{6} \mathrm{O}\right)_{2}\right]$ was obtained from Aldrich and was used as received.

Gels were made from 5-methylresorcinol (MR) and formaldehyde $(\mathrm{F})$ in water $(\mathrm{W})$ and then dried using supercritical $\mathrm{CO}_{2}$. After pyrolysis, the carbon aerogels prepared are characterized by high specific surfaces (over $500 \mathrm{~m}^{2} / \mathrm{g}$ ), low densities (below $0.2 \mathrm{~g} / \mathrm{cm}^{3}$ ) and tailorable porosity (from $40 \%$ to over $90 \%$ microporosity).

The composition and morphology of the carbon aerogels was determined using an EDAX JEOL 6042 analyzer and Leo Supra 35 Scanning Electron Microscope (SEM).

For nitrogen adsorption analyses, a Sorptometer KELVIN 1042 built by Costech International was used. The gas volume $\left(\mathrm{N}_{2}\right)$ adsorbed on the surface of materials at liquid nitrogen temperature was measured with a measurement accuracy of over $97 \%$. Helium was used as a carrier gas with nitrogen being the adsorptive gas. Brunauer-Emmet-Teller (BET) specific surface areas $\left(\mathrm{S}_{\mathrm{BET}}\right)$ were calculated according to the BET theory, specific micropore volumes $\left(\mathrm{V}_{\text {mic }}\right)$ from t-plot theory and pore size distributions calculated using Barrett-Johner-Halendar (BJH) theory.

For the thermogravimetrics analysis a SETARAM LabSys 2000 TG/DTA equipment was used. Experiments were performed in the $\mathrm{N}_{2}(99.999 \%)$ flow $-100 \mathrm{ml} / \mathrm{min}$ - at a heating rate of $10 \mathrm{deg} / \mathrm{min}$ in a Pt crucible. The sample mass was $8.5 \mathrm{mg}$.

The activation of the gels was carried out by placing the carbon aerogel in a tubular furnace under a flow of the activating agent. The temperature was risen from the room temperature to $1173 \mathrm{~K}$ in two hours and then it was held at $1173 \mathrm{~K}$ for a certain time as described in Table 3 . Finally, the oven was cooled down under its own thermal mass.

\section{Results and discussion}

The aerogels were prepared from the local industrial by-product 5-methylresorcinol by further drying with supercritical $\mathrm{CO}_{2}$. They underwent pyrolysis according to an improved treatment developed by our group.

In the earlier studies the temperature program was linear with rate $10^{\circ} \mathrm{C} / \mathrm{min}$ starting from the room temperature to the final temperature $1273 \mathrm{~K}$, staying at $1273 \mathrm{~K}$ for 2 hours. Thermogravimetric analysis (TGA) 
allowed a very useful evaluation of the temperature program used for the pyrolysis.

Knowing the mass loss at different temperatures obtained from thermogravimetric analysis (see TGA curve in Fig. 1) it was possible to optimize the pyrolysis temperature program basing on minimal evolution of degradation products resulted from slowing down the program rate for certain temperature intervals and taking into consideration exothermic nature of reactions at temperatures around $570 \mathrm{~K}$. It appeared that there is no need for that high final temperature also using the proposed program, because the change in mass was not noticeable at higher temperatures.

Specific surface areas of gels pyrolysed using the refined program allowed favorable comparison with the results obtained earlier by our group. It is clearly seen that the improvements made to the pyrolysis program had a significant positive impact on results: the gels produced via the new program are of increased porosity, and the surface areas sometimes twice as high as those obtained via the original non optimized programs.

The size of the pores and particles vary according to the molar ratios of 5-methylresorcinol to catalyst $(\mathrm{MR} / \mathrm{C})$ and water to 5-methylresorcinol

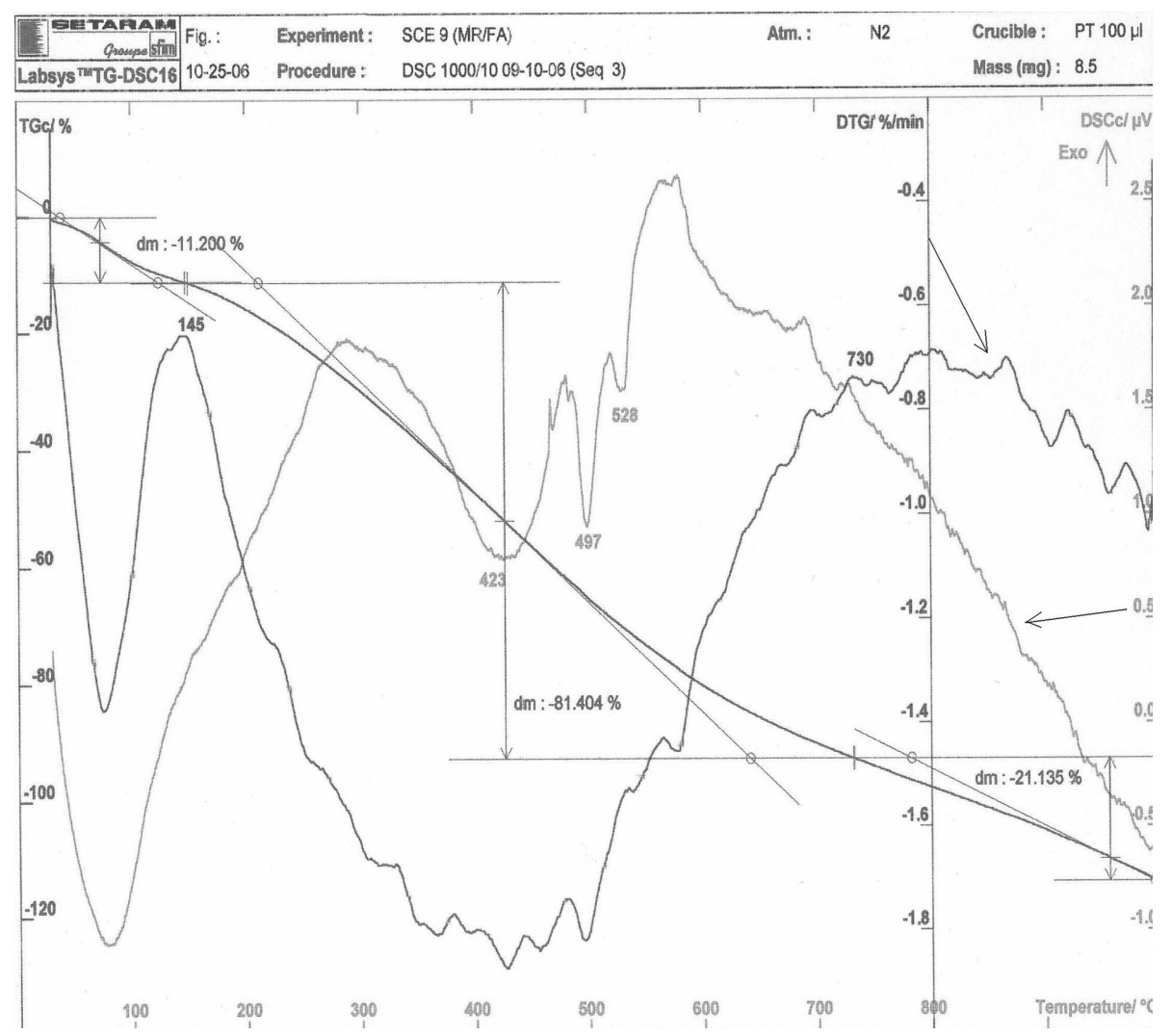

Fig. 1. Typical thermogram for a 5-methylresorcinol-formaldehyde carbon aerogel. 
(W/MR). Figures $2 \mathrm{a}$ and $2 \mathrm{~b}$ show the difference in particle size in two carbon aerogel samples prepared using different reagent concentrations; the size of the pores is varying according to the molar ratios MR/C and W/MR, too. Thus, carbon aerogel prepared using MR/C and W/MR ratios of 92 and 44 respectively, pyrolysed at $1273 \mathrm{~K}$ (Fig. 2a) presents a higher particle size and a lower microporosity than aerogel (Fig. 2b), which is prepared with molar ratios of MR/C and W/MR equal to 30 and 24 respectively and pyrolysed at $973 \mathrm{~K}$. The capability of tailoring the particle size and specific surface area is of great interest in order to prepare carrier specifically according to the necessities and properties of the compound to be impregnated in it.

Table 1 shows the surface areas and pore volumes of gels pyrolysed using common temperature programs and the program prepared after carrying out the thermometric study, allowing their comparison. The Langmuir specific surface areas obtained using the new temperature program were higher than those obtained via the non optimized programs, in the case of the MR/C $=60$ gel more than two times greater. The porosity was also considerably enhanced as can be seen from the micropore and total pore volumes.

$\mathrm{CO}_{2}$ activation process is an effective way to introduce micropores in carbon aerogels (CA), which is enhanced with the increase of activation time. CA has a network structure of primary carbon particles, providing predominant mesopores. These carbon particles have a few micropores relatively to their precursory organic aerogels, which have nearly no micropores. Activated carbon aerogels (ACA) had a bimodal pore size distribution of micropores and mesopores. Activation treatment invites more micropores than carbonization process. Such an activation treatment does not change the basic network structure, so that ACA has both abundant micropores and mesopores. It must be taken into account that weight loss during the activation is significant.

a)

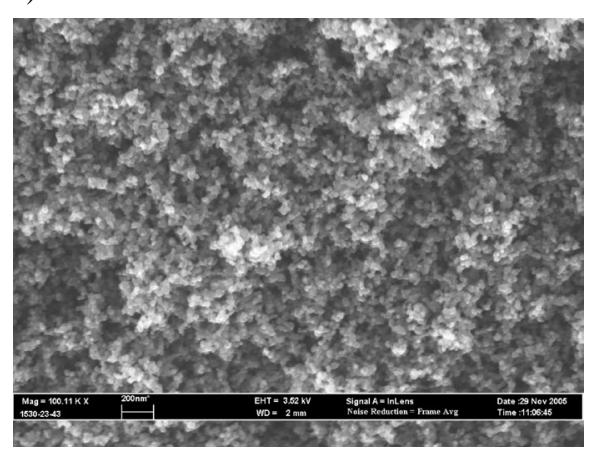

b)

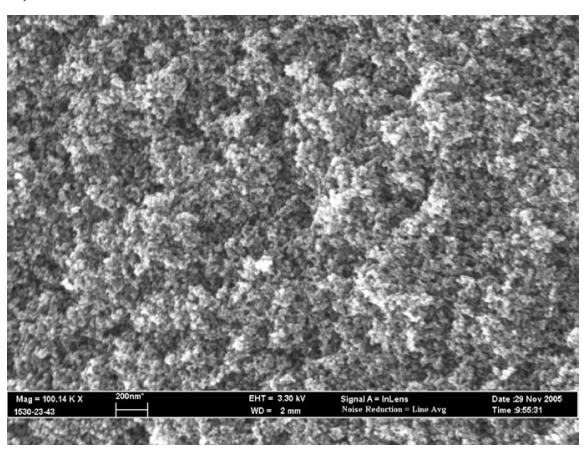

Fig. 2. Pictures of 5-methylresorcinol-formaldehyde carbon aerogels showing the dependence of the pore size on the composition and temperature of pyrolysis. a) $\mathrm{MR} / \mathrm{C}=92, \mathrm{~W} / \mathrm{MR}=44, \mathrm{MR} / \mathrm{F}=0.5,1273 \mathrm{~K}$ pyrolysis. b) $\mathrm{MR} / \mathrm{C}=30, \mathrm{~W} / \mathrm{MR}=$ $24, \mathrm{MR} / \mathrm{F}=0.5$, pyrolysed at $973 \mathrm{~K}$. (MR/F is the molar ratio of 5-methylresorcinol to formaldehyde). 
Table 1. Data on pyrolysis using the optimized program at $1053 \mathrm{~K}$ and other common temperature programs for some MR-F gels

\begin{tabular}{|c|c|c|c|c|c|}
\hline Pyrolysis & $\mathrm{MR} / \mathrm{C}$ & W/MR & $\begin{array}{c}\text { Langmuir } \\
\text { surface area, } \\
\mathrm{m}^{2} / \mathrm{g}\end{array}$ & $\begin{array}{l}\text { Mic. V, } \\
\mathrm{mm}^{3} / \mathrm{g}\end{array}$ & $\begin{array}{l}\text { Total pore volume, } \\
\mathrm{mm}^{3} / \mathrm{g}\end{array}$ \\
\hline $\begin{array}{l}\text { Optimised pyrolysis at } \\
1053 \mathrm{~K}\end{array}$ & 60 & 45 & 790 & 120 & 640 \\
\hline Non pyrolysed sample & 60 & 45 & 390 & - & 441 \\
\hline $\begin{array}{l}\text { Non-optimised } \\
\text { program at } 1273 \mathrm{~K}\end{array}$ & 60 & 45 & 380 & 45 & na \\
\hline $\begin{array}{l}\text { Optimised pyrolysis at } \\
1053 \mathrm{~K}\end{array}$ & 90 & 45 & 700 & 160 & 360 \\
\hline Non pyrolysed sample & 90 & 45 & 170 & - & 200 \\
\hline $\begin{array}{l}\text { Non-optimised } \\
\text { program at } 1053 \mathrm{~K}\end{array}$ & 90 & 45 & 640 & 140 & na \\
\hline $\begin{array}{l}\text { Non-optimised } \\
\text { program at } 1273 \mathrm{~K}\end{array}$ & 90 & 45 & 540 & 88 & na \\
\hline
\end{tabular}

na $=$ not available

The properties of the samples activated by $\mathrm{CO}_{2}$ and $\mathrm{H}_{2} \mathrm{O}$ are shown in Table 2 .

In Figures $3 \mathrm{a}$ and $3 \mathrm{~b}$, the behavior of the activation is analyzed, $\mathrm{CO}_{2}$ and $\mathrm{H}_{2} \mathrm{O}$ being the activating agents. Figure $3 \mathrm{a}$ shows the percentage of microporosity of the samples which reaches over $85 \%$ for $\mathrm{CO}_{2}$ activation and over $70 \%$ for $\mathrm{H}_{2} \mathrm{O}$ activation. It is important to notice that he final porosity of the sample can be adjusted according to the activation time, thus, samples can be obtained with microporosity ranging from below $40 \%$ to over $80 \%$. Figure $3 \mathrm{~b}$ shows the BET specific surface area of carbon aerogels after

Table 2. Data on activation of carbon aerogels using $\mathrm{CO}_{2}$ and $\mathrm{H}_{2} \mathrm{O}$ as activating agents. All samples were pyrolysed at $1053 \mathrm{~K}$

\begin{tabular}{|c|c|c|c|c|c|c|c|c|c|c|}
\hline Sample & $\begin{array}{c}\text { Act. } \\
\text { agent }\end{array}$ & $\mathrm{t} / \mathrm{h}$ & $\mathrm{V}$ mic & $\begin{array}{c}\text { Total } \\
\text { pore V }\end{array}$ & $\begin{array}{c}\text { Mic } \\
\text { area }\end{array}$ & $\begin{array}{c}\text { Non-Mic } \\
\text { area }\end{array}$ & S BET & $\%$ Mic & $\begin{array}{c}\text { Density, } \\
\mathrm{g} / \mathrm{mL}\end{array}$ & $\begin{array}{c}\text { Mass lost, } \\
\%\end{array}$ \\
\hline Dried & - & - & 0 & nd & 0 & 295.48 & 295.5 & 0 & 0.2061 & - \\
Blank & - & 0 & 82.92 & nd & 235.34 & 240.18 & 475.5 & 49.49 & 0.303 & 0 \\
$\mathrm{H}_{2} 1_{1}$ & $\mathrm{H}_{2} \mathrm{O}$ & 0.5 & 137.0 & 903.12 & 388.78 & 324.07 & 712.9 & 54.54 & 0.307 & 24.46 \\
$\mathrm{H}_{2} 0_{2}$ & $\mathrm{H}_{2} \mathrm{O}$ & 2 & 198.5 & nd & 563.21 & 207.39 & 770.6 & 73.09 & 0.311 & 25.09 \\
$\mathrm{H}_{2} 03$ & $\mathrm{H}_{2} \mathrm{O}$ & 4 & 149.4 & 876.25 & 423.96 & 257.93 & 681.9 & 62.172 & 0.287 & 42.99 \\
$\mathrm{CO}_{2} 1$ & $\mathrm{CO}_{2}$ & 0.5 & 263.2 & 812.91 & 746.96 & 281.32 & 1028.2 & 72.64 & 0.245 & 27.33 \\
$\mathrm{CO}_{2} 2$ & $\mathrm{CO}_{2}$ & 1 & 290.7 & nd & 825.11 & 144.30 & 969.4 & 85.11 & 0.217 & 33.40 \\
$\mathrm{CO}_{2} 3$ & $\mathrm{CO}_{2}$ & 1.5 & 472.4 & nd & 1340.9 & 248.92 & 1589.8 & 84.34 & 0.178 & 42.72 \\
$\mathrm{CO}_{2} 4$ & $\mathrm{CO}_{2}$ & 2 & 261.2 & 1253.3 & 741.1 & 914.63 & 1655.8 & 44.76 & 0.158 & 66.92 \\
\hline
\end{tabular}

The column "Act. agent" shows the gas flowed through the reactor during the activation process. $\mathrm{t} / \mathrm{h}$ is the time of activation in hours. V. mic is the volume of micropores of the samples in $\mathrm{mm}^{3} / \mathrm{g}$. Total pore $\mathrm{V}$ is the total pore volume in $\mathrm{mm}^{3} / \mathrm{g}$. Mic area and Non-Mic area are the microporous and non-microporous areas respectively, in $\mathrm{m}^{2} / \mathrm{g}$. S BET is the BET specific surface area in $\mathrm{m}^{2} / \mathrm{g}$. \% Mic is the percentage of microporosity in the sample. 
a)

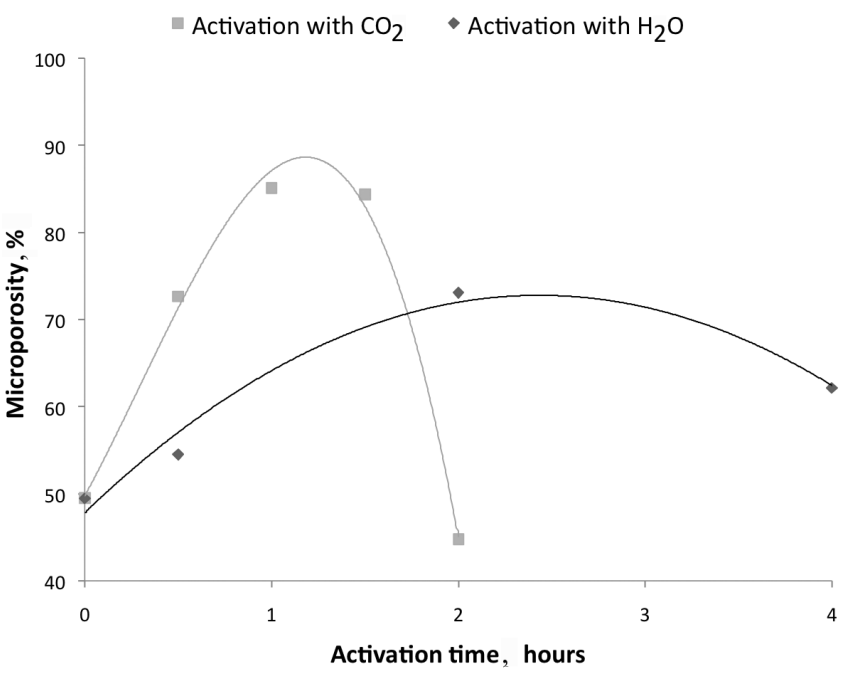

b)

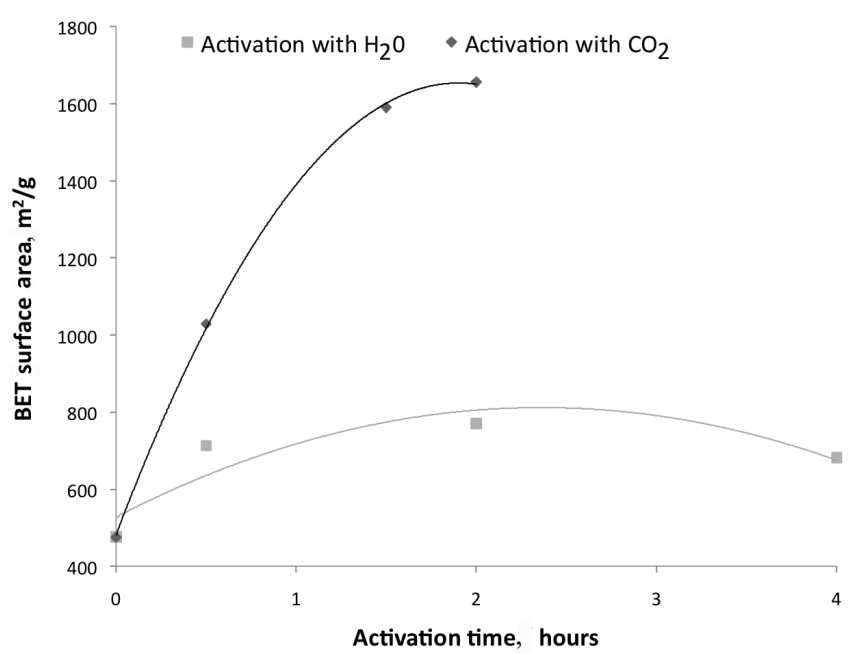

Fig. 3. Microporosity (3a) and BET area (3b) of activated carbon aerogels activated using $\mathrm{CO}_{2}$ and $\mathrm{H}_{2} \mathrm{O}$.

activation. After two hours of activation with $\mathrm{CO}_{2}$, a maximum seems to be reached as the value is not growing and the loss in the mass of the carbon aerogels does not allow measurements over the time studied. In the case of $\mathrm{H}_{2} \mathrm{O}$ activation, no important changes in the specific surface area with the activation after a 4 hours study were observed.

The next step was the impregnation of carbon aerogel obtained. In regard to the impregnation, the use of $\mathrm{scCO}_{2}$ as a carrier of the palladium complex into the pores is facilitated by the lack of solvent's surface tension. The 
solubility of the metal complex in $\mathrm{scCO}_{2}$ was crucial in order to allow metals enter the carbon network and deposit in the porous after reduction. Metal complexes are typically insoluble in $\mathrm{scCO}_{2}$ but by employing the fluorous complex $\left[\mathrm{Pd}\left(\mathrm{C}_{4} \mathrm{HF}_{6} \mathrm{O}\right)_{2}\right]$, which showed sufficient solubility, this difficulty was overcome. Thus, the study of the deposition of many other metals through this process is now possible, and interesting catalytic properties can now be studied and tested.

Figure 4 shows the composition of the core of the carbon aerogel after being impregnated with palladium nanoparticles. With the decompression in the reaction after reducing the $\mathrm{Pd}(\mathrm{II})$ complex, the particles of black Pd deposited all around the walls of the reactor as well as on the surface of the carbon aerogel. In order to carry out the analysis of the surface, the thin layer of black Pd formed was polished out.

The homogeneous distribution of black Pd (white dots) in the core of the carbon aerogel at two different scales can be observed in Fig. 5. On the left, a sensitive to metal atoms Back Scatter Electron (BSE) detector is applied, and one can see how the particles of Pd occupy the inner of the carbon aerogel homogeneously. In Fig. 5 at the right side the same image is shown with no BSE detector applied. A homogeneous distribution of the catalyst in the carrier is a key fact in catalysis.

In our research, it was also found that after the impregnation process, the specific surface area, as well as the pore volume decrease. In Table 3 two gels with different composition are compared before and after impregnation with palladium: for the gel of composition $M R / C=60$, a diminution in the

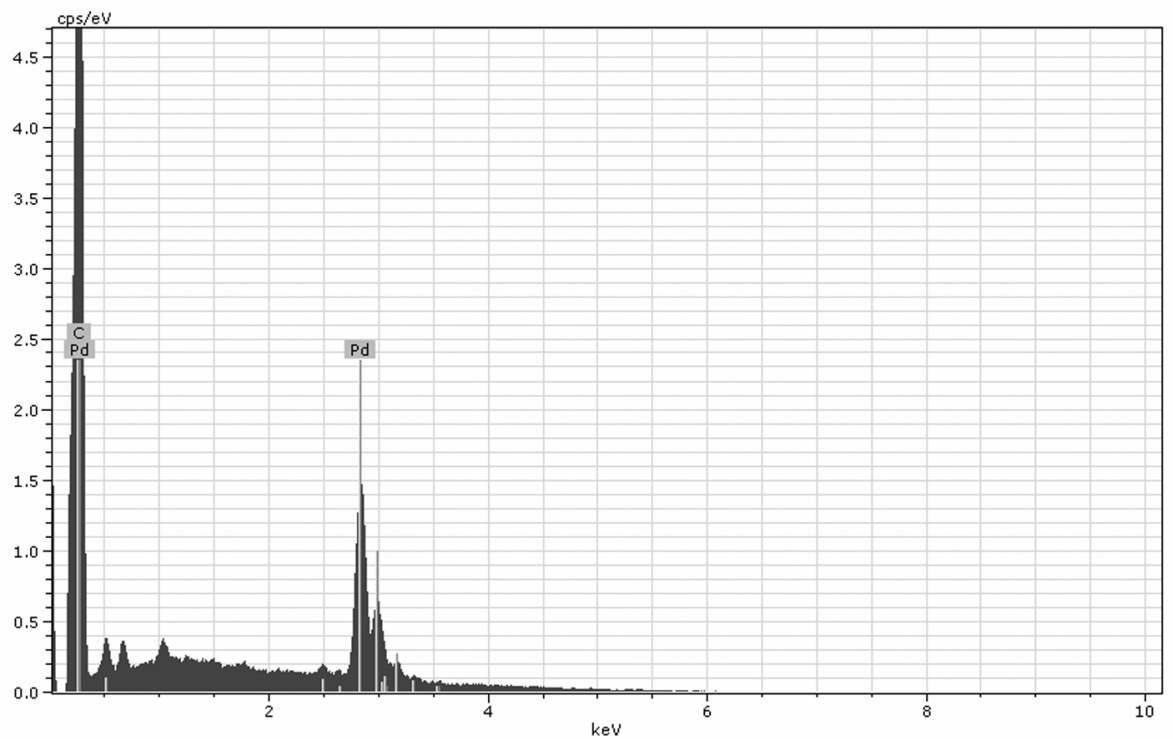

Fig. 4. Analysis of the composition of the core of carbon aerogels after impregnation with Pd nanoparticles. 


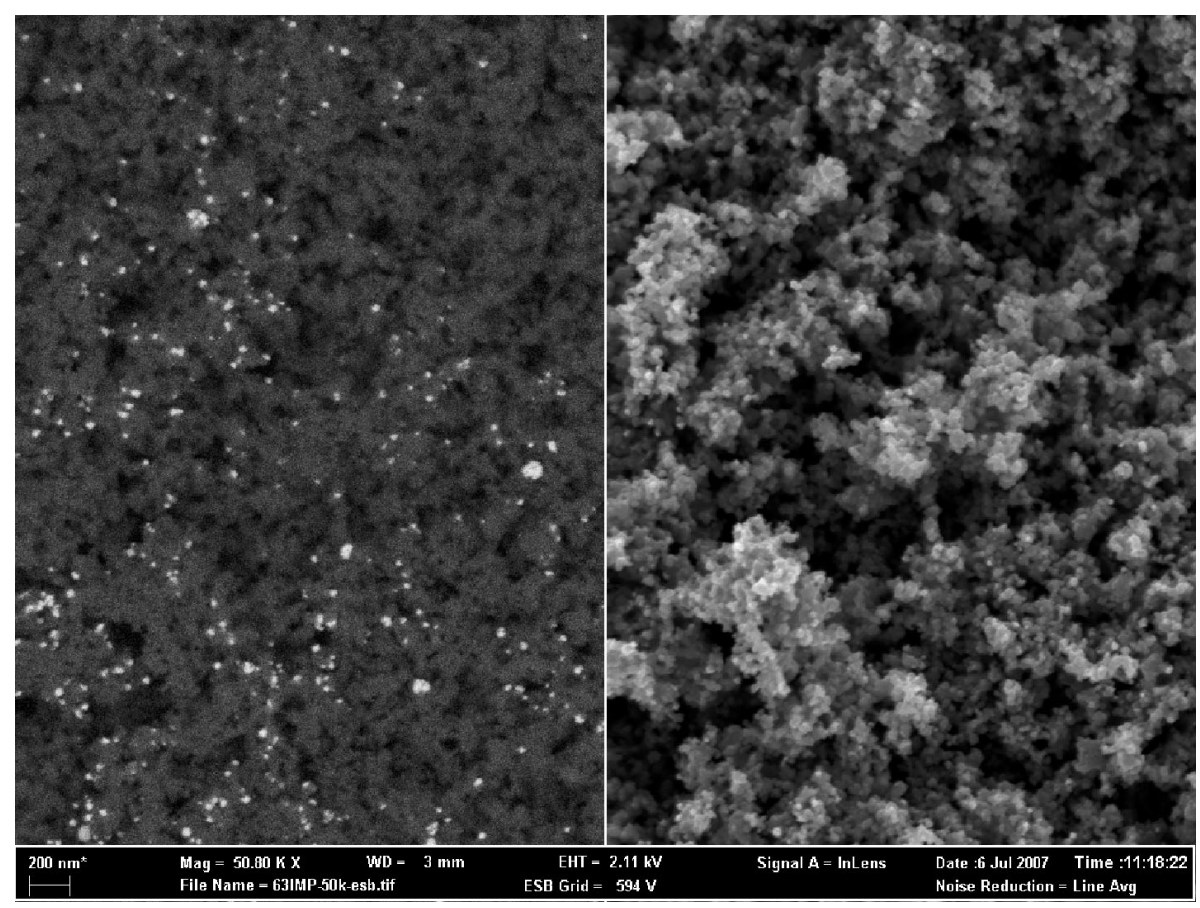

Fig. 5. Left, SEM picture taken using a Back Scattering Electron (ESB) detector which shows the particles of Pd as white dots. Right: the same region of the sample without ESB detector.

Table 3. $\mathbf{N}_{\mathbf{2}}$-adsorption data for aerogels with differing amounts of catalyst

\begin{tabular}{|c|c|c|c|c|c|}
\hline MR/C & W/MR & & $\begin{array}{c}\text { Langmuir surface } \\
\text { area, } \mathrm{m}^{2} / \mathrm{g}\end{array}$ & $\begin{array}{c}\text { Pore vol., } \\
\mathrm{mm}^{3} / \mathrm{g}\end{array}$ & Mic. vol., $\mathrm{mm}^{3} / \mathrm{g}$ \\
\hline 60 & 45 & N.Imp. & 790 & 640 & 120 \\
60 & 45 & Imp. & 660 & 620 & 70 \\
90 & 45 & N.Imp. & 696 & 360 & 160 \\
90 & 45 & Imp. & 695 & 345 & 115 \\
\hline
\end{tabular}

Aerogels impregnated (Imp.) are compared to those non-impregnated (N. Imp.). MR/C refers to the molar ratio 5-methylresorcinol to catalyst and W/MR water to 5-methylresorcinol. "Pore vol." is the total volume of pores and "Mic. vol" is the volume of micropores.

Langmuir specific surface area of over $15 \%$ and in the micropore volume of over $40 \%$ was observed, indicative of the size of the palladium particles that remained within the network. However, for the gel of composition MR/C = 90, there were no significant changes in the total Langmuir specific surface area whilst micropore volume decreased almost $30 \%$ after Pd impregnation. A more detailed study on the effects of impregnation on the specific surface area is in progress. 
The palladium-coated support potentially possesses interesting applications in heterogeneous catalysis, for example, in continuous-flow palladium catalyzed redox reactions employing a supercritical mobile phase, making the products of this work of interest for further study with a view to their industrial application in such processes. The method here demonstrated for support impregnation with palladium is also potentially adaptable for a range of other metal catalysts, facilitating production of supported catalysts for a variety of catalytic transformations.

The resulting carbon aerogel with palladium nanoparticles was used for hydrogenation of cyclo-octene. The first brief results demonstrated that this kind of catalyst worked well with high yield during several cycles, but a more detailed study is needed for full description of this kind of catalysts.

\section{Conclusions}

The process of preparation of carbon aerogel used for impregnation and activation was optimized in sense of pyrolysis program. It was based on the thermogravimetrics study of organic aerogels, which allowed us to modify the pyrolysis program leading to significantly higher values of specific surface areas and total pore volumes.

Carbon aerogels obtained were used to prepare porous carbon material with metal nanoparticles deposited in it. A method for the impregnation of metals in carbon aerogels and further reduction of metal complex to metal was developed.

The process was quick, simple and produced excellent results, with the metal evenly deposited across the surface and inside the pores. Palladium deposited inside the microporous structure of the gels decreased, as expected, the pore size of the samples as well as the total microporosity.

Prior to impregnation, the activation of carbon aerogels was proven to be a successful method to increase the specific surface area as well as the porosity. These properties can be adjusted to the desired value according to the time for which the activation process is carried out.

\section{Acknowledgements}

This work was supported by the European Commission according to the "SuperGreenChem" EC Contract No. MRTN-2003-504005.

Authors express their thanks to Olga Volobujeva for making analysis of the materials prepared during this study. 


\section{REFERENCES}

1. Tamon, H., Ishizaka, H. Porous characterization of carbon aerogels // Carbon. 1998. Vol. 36, Issue 9. P. 1397-1409.

2. Pekala, $R$. W. Organic aerogels from the polycondensation of resorcinol with formaldehyde // J. Mater. Sci. 1998. Vol. 24. P. 3221-3227.

3. Pekala, R. W., Alviso, C. T. Carbon aerogels and xerogels // Mater. Res. Soc. Symp. Proc. 1992. Vol. 270. P. 3-14.

4. Pérez-Caballero, F., Peikolainen, A.-L., Uibu, M., Kuusik, R., Volobujeva, O., Koel, M. Preparation of carbon aerogels from 5-methylresorcinol-formaldehyde gels // Micropor. Mesopor. Mater. 2008. Vol. 108, Issues 1-3. P. 230-236.

5. Peikolainen, A-L., Pérez-Caballero, F., Koel, M. Low-density organic aerogels from oil shale by-product 5-methylresorcinol // Oil Shale. 2008. Vol. 25, No. 3. P. 348-358.

6. Baumann, T.F., Satcher, J. H. Jr. Template-directed synthesis of periodic macroporous organic and carbon aerogels // J. Non-Cryst. Solids. 2004. Vol. 350. P. 120-125.

7. Tamon, H., Ishizaka, H., Araki, T., Okazaki, M. Control of mesoporous structure of organic and carbon aerogels // Carbon. 1998. Vol. 36, Issue 9. P. 1257-1262.

8. Tamon, H., Ishizaka, H., Mikam, M., Okazaki, M. Porous structure of organic and carbon aerogels synthesized by sol-gel polycondensation of resorcinol with formaldehyde // Carbon. 1997. Vol. 35, Issue 6. P. 791-796.

9. Yoshizawa, N., Hatori, H., Soneda, Y., Hanzawa, Y., Kaneko, K. Dresselhaus, M.S. Structure and electrochemical properties of carbon aerogels polymerized in the presence of $\mathrm{Cu}^{2}+/ /$ J. Non-Cryst. Solids. 2003. Vol. 330, Issues 1-3. 99-105.

10. Baumann, T. F., Worsley, M. A., Yong-Jin Han, T., Satcher Jr, J. H. High surface area carbon aerogel monoliths with hierarchical porosity // J. Non-Cryst. Solids. 2008. Vol. 354, Issue 29. 3513-3515.

11. Wang, J., Yang, X., Wu, D., Fu, R., Dresselhaus, M. S., Dresselhaus, G. The porous structures of activated carbon aerogels and their effects on electrochemical properties // J. Power Sources. 2008. Vol. 185, Issue 1. P. 589-594.

12. Moreno-Castilla, C. Maldonado-Hódar, F. J. Carbon aerogels for catalysis applications: An overview // Carbon. 2005. Vol. 43, Issue 3. P. 455-465.

13. Cotet, L. C., Gich, M., Roig, A., Popescu, I. C., Cosoveanu, V., Molins, E., Danciu. $V$. Synthesis and structural characteristics of carbon aerogels with a high content of Fe, Co, Ni, Cu, and Pd // J. Non-Cryst. Solids. 2006. Vol. 352, Issues 26-27. P. 2772-2777.

14. Cacchi, S., Cotet, C. L., Fabrizi, G., Forte, G., Goggiamani, A., Martn, L., Martnez, S., Molins, E., Moreno-Maas, M., Petrucci, F., Roig, A., Vallribera, A. Efficient hydroxycarbonylation of aryl iodides using recoverable and reusable carbon aerogels doped with palladium nanoparticles as catalyst // Tetrahedron. 2007. Vol. 63, Issue 11. P. 2519-2523.

15. Saquing, C. D., Kang Dafei, Aindow, M., Erkey, C. Investigation of the supercritical deposition of platinum nanoparticles into carbon aerogels // Micropor. Mesopor. Mater. 2005. Vol. 80, Issues 1-3. P. 11-23.

16. Zhang Ying, Erkey, $C$. Preparation of supported metallic nanoparticles using supercritical fluids: A review // J. Supercrit. Fluid. 2006. Vol. 38, Issue 2. P. 252-267. 
17. Wood, C. D., Tan Bien, Zhang Haifei, Cooper, A. I. Supercritical Carbon Dioxide as a Green Solvent for Polymer Synthesis // Thermodynamics, Solubility and Environmental Issues / T. V. Letcher (ed.). Elsevier, 2007. P. 383-396.

Presented by V. Oja

Received January 7, 2008 